MATEC Web of Conferences 31, 06001 (2015)

DOI: $10.1051 /$ matecconf/ 20153106001

(C) Owned by the authors, published by EDP Sciences, 2015

\title{
Review of Research on Carbon-free Car
}

\author{
WANG Zeng-sheng ${ }^{1, a}$, YANG Han-song ${ }^{1}$, KONG Ling-yun ${ }^{1}$ \\ ${ }^{1}$ Huanghe Science and Technology College ,Zhengzhou,450063
}

\begin{abstract}
. studied comprehensively from the scheme design and innovative design, mathematical modeling, research methods and other aspects of carbon-free car, discussed the research content and distribution characteristics of literature, summed up the general scheme and innovative design of carbon-free car, It provides a certain reference value for the design and research of carbon-free car.
\end{abstract}

\section{Introduction}

"Carbon-free car" is one of the national college engineering comprehensive ability training contest theme, the design requirements of a gravity potential driving direction control function car, the game requires the car to $\mathrm{S}$ shape and figure 8 around the obstacle. Since the competition, teachers and students in many colleges and universities begin to study the car without carbon, and published a series of research papers.

The schematic diagram of the car is shown in Figure 1, and the energy of its driving and turning is based on the energy conversion principle, by a given gravitational potential energy is converted obtained. The given gravity potential energy is supplied by the standard weight of $1 \mathrm{Kg}$, The drop height of the weight is $400+2 \mathrm{~mm}$. The standard weight is always carried by the trolley, and it is not allowed to fall from the car.

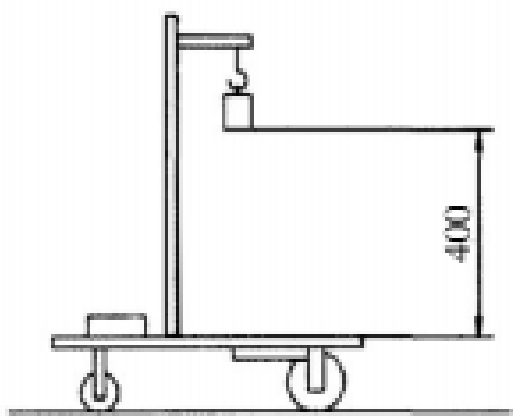

Figure 1. Schematic diagram of carbon- free car

In the "S" type track game, produce a "S" type obstacle track spacing values within the range of $700-1300 \mathrm{~mm}$. The competition car can automatically bypass the obstacle when the track is on the line, as shown in Figure 2 . The obstacle is the round rod of the diameter of $20 \mathrm{~mm}$, high $200 \mathrm{~mm}$, placed equidistant along the track center line. Track width of 2 meters, starting from the end of the

\footnotetext{
a Corresponding author: 93611898@qq.com
}

spacing between the first obstacle and the obstacles and barriers are the same. The competition results are determined by the distance of the car and the number of successful bypassing obstacles.

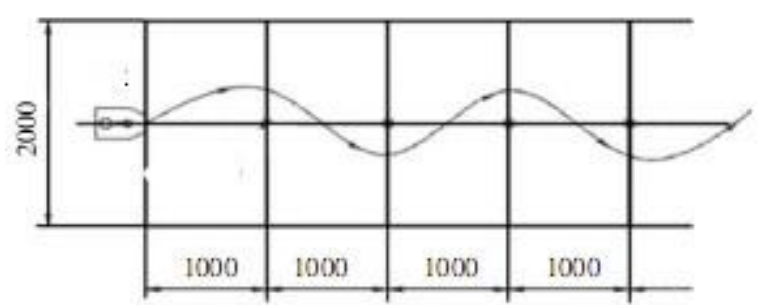

Figure 2. the car around the S shaped track running

In the "8" shape track, the car running around two obstacles according to the figure 8 in half sheets of standard table tennis table (length $1525 \mathrm{~mm}$, width $1370 \mathrm{~mm}$ ) Obstacle diameter of $20 \mathrm{~mm}$, length of $200 \mathrm{~mm}$ 2 rod, placed some distance apart in half a sheet of standard table tennis table in the line, the distance is within the range of $300 \sim 500 \mathrm{~mm}$. The final result depends on how many laps the car is accomplished, as shown in Figure 3.

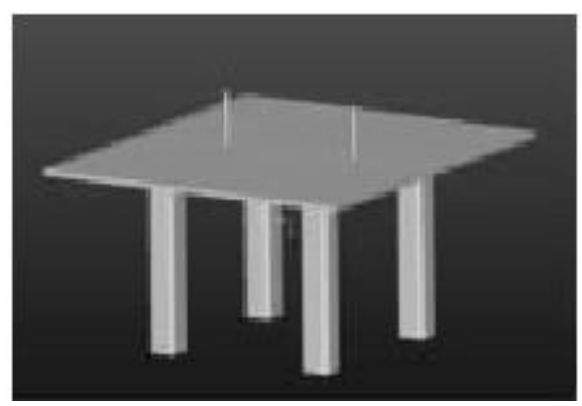

Figure 3. Ping-pong table and the obstacles set Figure

From the analysis of the number of documents: the study of S car obstacle avoidance research for more than 
8 "car obstacle avoidance, the main reason is the figure 8 car is new projects. From the specific content of the literature, there are three main categories, namely general solution of car, steering and transmission system research, study on the steering system, a considerable amount of literature on the three aspects. According to the literature research, will be discussed from the following aspects.

\section{Research and innovation design of the scheme}

By functional analysis of the car, the car needs to complete the conversion of gravitational potential energy, drive, walk itself automatically avoid obstacles. To facilitate the design of this car to be completed according to the function of the modular design of the car is divided into six parts (frame, original mechanism, transmission, steering mechanism, running gear, fine adjustment mechanism). In order to obtain a satisfactory solution in extended thinking design of each module, to seek a variety of possible solutions and ideas. The function module of the car and implementation plans as shown in Figure 4.

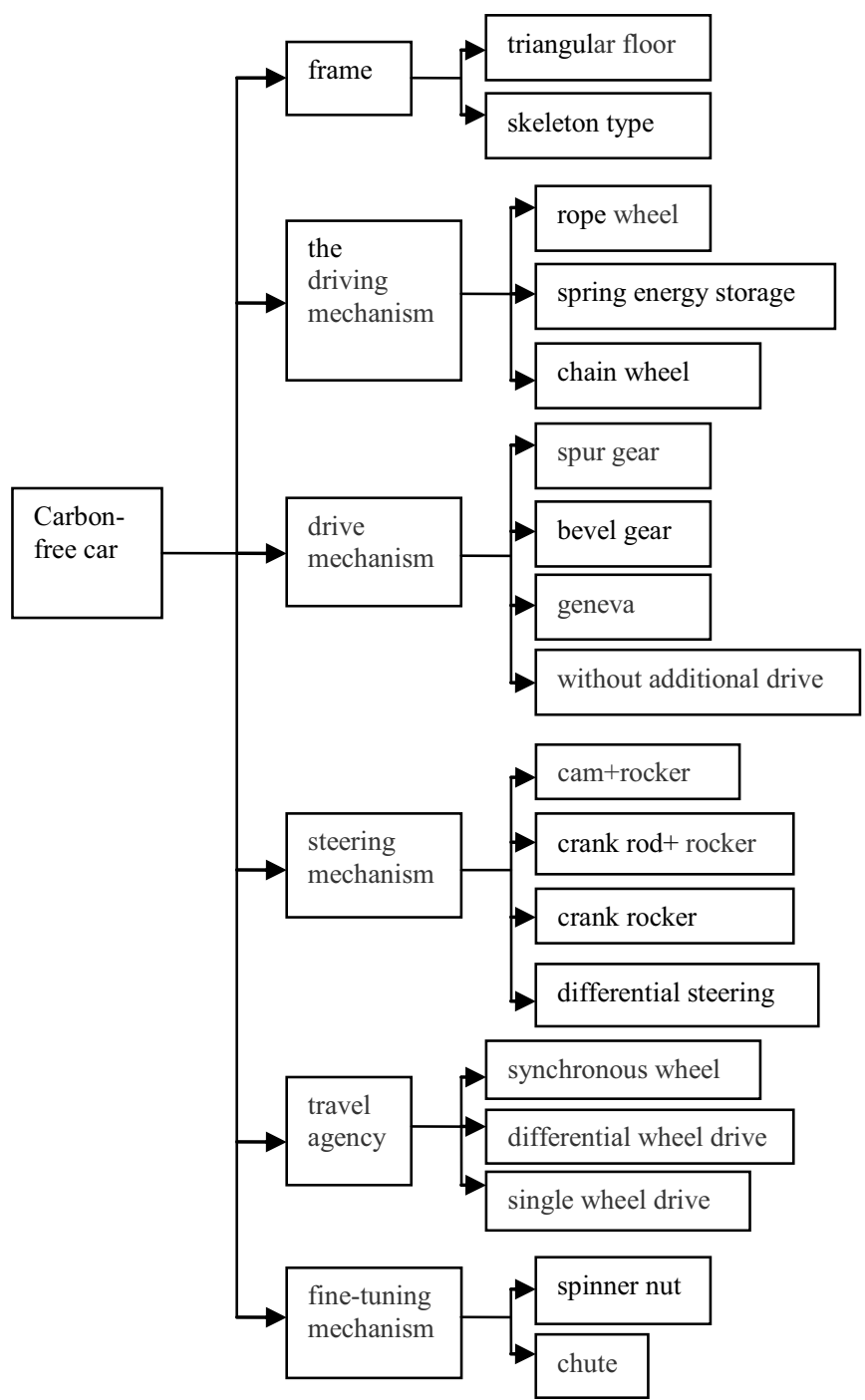

Figure 4. The function module of the car and implementation plans
From the analysis of specific programs: car driving mechanism commonly used rope wheel mechanism; transmission structure is widely used gear transmission for its high efficiency; driving mode is mainly driven by a single wheel steering mechanism; in the S- car more using the crank rocker mechanism, and in the 8 shaped car obstacle avoidance, mainly to achieve more based on incomplete gear and cam mechanism; adjustment mechanism mainly adopts trimming nut and sliding mechanism; in addition, some local programs have been used in innovative design, mainly:

Liu Run ${ }^{[1]}$ in order to restrict the front wheel steering angle, the spring constraint is added to the $\mathrm{S}$ type car steering mechanism,

Ji Yuan-jiang ${ }^{[2]}$ used the change gear as the driving mechanism of the $\mathrm{S}$ shape car, so that the formation of the speed difference driven moving by simple harmonic wave track wheel.

$\mathrm{Wu}$ Zhao-chun ${ }^{[3]}$ used a crank connecting rod mechanism as the guiding mechanism of the $\mathrm{S}$ shape car, and put the ordinary bearing and a one-way needle bearing with the use of rear wheel drive and ensure the implementation of alternative vehicle stability.

Ceng Si-long ${ }^{[4]}$ used slider crank steering mechanism in the front wheel of the $\mathrm{S}$ shape car for matching angle and the rear wheel steering speed, the characteristics of slider crank mechanism itself cleverly used, to avoid the car to the rear differential steering error.

Wen Wei-song ${ }^{[5]}$ had made a comparative analysis of the cam mechanism, to achieve car steering eccentric mechanism, crank rocker mechanism and transmission gear mechanism, synchronous belt mechanism and a chain wheel mechanism, the final selection of the crank rocker mechanism and sprocket mechanism respectively as steering and transmission mechanism of the S type car.

Zou Guang-ming ${ }^{[6]}$ used a scroll spring storage in the $\mathrm{S}$ shape car, the storage part of the gravitational potential energycan be transformed into a heavy block mechanism of elastic potential energy, and then converted to kinetic energy to drive the car, and reduce the falling speed and heavy block to make the car running smoothly role.

\section{Study on the mathematical model of the car}

Study on motion mathematical model in the literature are relatively few:

The document [1] established the transfer function model of the steering mechanism of the $\mathrm{S}$ shape car, and selected the spring coefficient, the steering angle and other parameters of the simulation, the simulation results and the experimental results are identical.

The document [2] established the motion equation of the $\mathrm{S}$ shape car which driven by change gear, and used MATLAB simulated trajectory.

Cao $\mathrm{Bin}^{[7]}$ derived the equations of motion of 8 shape car by geneva mechanism, and used the formula and iterative tracing point imaging function of Excel2007, correct simulation was realized and the parameters of the vehicle trajectory. 
Chen Hai-wei ${ }^{[8]}$ established a mathematical model of the $\mathrm{S}$ shape car which by linkage mechanism for its steering mechanism.

$\mathrm{Hu}$ Hong-ying ${ }^{[9]}$ completed the three $\mathrm{S}$ trolley track analysis, calculation, modeling and simulation, and to determine the influence of structure parameters on trajectory. The three cars are respectively by a crank guide rod, crank, cam slider to achieve.

Liu Guang [ ${ }^{10]}$ established a mathematical model of S shape car which by incomplete gear for its steering mechanism, and the trajectory simulation using maple.

Wang Jian-jun ${ }^{[11]}$ established the model of steering angle and steering mechanism of 8 shape car. This kind of car by the spatial crank rocker combined with incomplete gear as the steering mechanism.

\section{Analysis of research methods}

Research by means of literature from the point of view, a lot of literatures used three-dimensional modeling analysis software such as PROE, ADAMS, SolidWorks, mathematical analysis software such as MATLAB, maple, the modern design method and programming software $\mathrm{VC}++$, can play a multiplier effect. For example:

The document [10] drew the exact route of the $S$ track of the car based on incomplete gear for its steering mechanism, which used Maple programming.

Zhang Si-wei ${ }^{[12]}$ completed the analysis of kinematic simulation of the overall structure of the $\mathrm{S}$ shape car based on Pro/E, tested the mechanical structure of the interference and dead point, improved the efficiency and success rate of mechanical structure design.

Zhang Yu-hang ${ }^{[13]}$ adjusted the design parameters of the car based on MATLAB, in order to match the steering angle of the front wheel and the angle of the rear wheel, the results simulated trajectory can directly reflect whether the design meet the requirements, to optimize the design.

To solve the side-slip problem of carbon-free car, applies Adams software parametric modeling functions and simulation capabilities to detect the impact of the front wheel position of carbon-free car on the trajectory linearity, $\mathrm{Hu} \mathrm{Zeng}^{[14]}$ analyzed and compared the experimental data with Matlab software.

For a better S type trajectory of the carbon-free car, applies the parametric modeling capabilities and simulation capabilities of the ADAMS software to simulate the crank-rocker mechanism, sine mechanism and RSSR space four-bar linkage mechanism for carbonfree car steering mechanism. Wang Zheng ${ }^{[15]}$ Compared the simulation results and drew the conclusion that sine mechanism is the best direction-control mechanism for the carbon-free car through the relative parameter design.

The motion simulation for the overall structure of the $S$ type car was carried out on the application of SolidWorks by Dai Hai-yan ${ }^{[16]}$, Li Shi-wen ${ }^{[17]}$ through the use of cam design module SolidWorks toolbox plug-in, to achieved the rapid design of disc cam, avoided the tedious design process by using the analytical calculation method, also avoided wasteful duplication of resources and time for processing experiment.

\section{Research of other aspects}

The document [14] studied the layout of the S shape car driven by single wheel, and the results showed that the front wheel on the side of the drive wheels obtains better running track, and the coplanar for the front wheel and the drive wheel are more conducive to ensure the car frame centroid trajectory straightness.

Guo Gang ${ }^{[18]}$ used the contradiction matrix of TRIZ theory, determined the technical parameters of the 8 shape car through contradiction matrix, found the specific design principles of the invention to the carbon-free car.

Du Lei ${ }^{[19]}$ established the model of energy consumption of the car, studied the relationship between the car forward distance and friction coefficient, wheel radius, the weight of the car , and analyzed the stability of the car.

Zhao Peng-fei ${ }^{[20]}$ analyzed the error sources of 8 shape car, pointed out that the main error sources of system error, the parts design processing error and assembling error, and put forward the corresponding compensation measures.

Fan Su-fen ${ }^{[21]}$ took the NC machining of the carbonfree car axle for example, for small diameter stainless steel screw, and put forward some concrete countermeasures from the aspects of cutting tools, cutting parameters

and lubricant selection, preparation and detailed producti on method and auxiliary fixture processing code.

Material selection and processing of the car was not often mentioned in the literature, often said only a couple of words. The main body material of aluminum alloy, nylon, organic glass, steel plate, aluminum alloy wheel axle; organic glass, nylon, plastic etc.. The car manufacture method with CNC milling machine, line cutting, rapid prototyping, laser machining, EDM, machining center, etc.

\section{Conclusions}

From the above analysis, the contents of carbon-free car literatures were still concentrated in the determine of the argument and parameter scheme, the specific implementation of the car, and the theoretical analysis of mathematical model was relatively small. Other aspects, such as material and production, energy consumption, error was more rare. The research used of modern design, analysis, simulation and parameters of the car to determine the multiplier effect, and further research of the theory on carbon-free car, but also need to be further strengthened.

\section{Thanks}

Fund project of key laboratory of industrial robot vision and control of Zhengzhou (project code: 121PYFZX179)

\section{References}

1. Liu Run, Zhu Xian-yong, Li Zhi-dong. Modeling and simulation for steering mechanism with spring 
constraint on energy-saving vehicle[J]. Tournal of machine design.2013,30 (9): 24-27.

2. .Ji Yuan-jin, Ren Li-hui, Gu Jian. Innovational structure design of carbon-free car by using centerchanged gear technique [J].Tournal of machine design 2014,03:71-74.

3. Wu Zhao-chun. Mechanism and motion analysis of carbon-free car [J].electronic production, 2013, (13): 36-36.

4. Zeng Si-long, Wen Yu, Ni Bai-yang. The innovative design of the steering mechanism of a car without carbon [J]. Silicon Valley, 2013,6 (10): 36-37.

5. Wen Wei-song, Liang Qian, Wang Qiang, Bai Qianwei. Optimization design of a carbon free car [J]. technology and business, 2014,05:283.

6. Zou Guang-ming, Yang Xiu-guang, Huang Chuan et al. Research on the potential energy-driven car with energy stored by vortex coil spring $[\mathrm{J}]$. Tournal of machine design,2012,29 (4): 32-35.

7. Cao Bin, Zhang Hai-bo, Zhu Hua-bing et al. Design of carbon-free car based on Geneva mechanism driving in 8-shaped track [J]. Journal of HeFei University of Technology (NATURAL SCIENCE EDITION), 2014, (6): 661-665704.

8. Chen Hai-wei, Zhang Qiu-ju, fan Shengyao. Mathematical Modeling:An Important Step in Engineering Training of College Students [J]. Journal of Wuxi Institute of Technology, 2011, 10 (4): 70-72.

9. Hu Hong-ying, Yu Jin-pu, Bao Er. Structure Design and Analysis of Carbon-free Cars [J]. Journal of Dalian Nationalities University, 2013,15 (5):500-504.

10. Liu Guang, Cao Kai, Liu Jian-qiao. Design of energy-saving car $[\mathrm{J}]$. Technological Development of Enterprise (Academic Edition), 2011,30 (7): 103111

11. Wang Jian-jun, Zhu Hai-long, Yin Hong-you. Establishment of Steering Control Device Mathematical Model of Carbon-free Trolley [J] Mechanotronics., 2014,20 (2): 21-23.

12. Zhang Si-wei, Huang Jian-tai, Lin Xiong-rui. Design of "Non-carbon Small Car"[J]. Automotive Engineers, 2013, (6): 33-35,54.

13. Zhang Yu-hang, Huang Li, Wang Kai et al. The design of steering system for 8 words around the barrier carbon-free car [J]. science and Technology Innovation Herald, 2014, (13): 88-89.

14. Hu Zeng, He Guo-qi, Wang Zheng et al. Design of Wheel Arrangement of Single-Drive Carbon-Free Car Based on Adams Software [J].Journal of Hunan University of Technology, 2013,27 (4): 53-56.

15. Wang, where the national flag, $\mathrm{Hu}$ Zeng et al. ADAMS software based carbon free car steering mechanism design [J]. Journal of Hunan University of Technology, 2013,27 (5): 28-32.

16. Dai Hai-yan, Cai Kai-wen, Wu Yue-lang. Design of Carbon-free Car Based on Engineering Training [J]. auto parts, 2014, (11): 26-27,37.

17. Li Shi-wen, Zhao Hai-long, He Xin. parametric design of cam for carbon free car based on SolidWorks Toolbox's electronic production,2014, (11): 50-51.
18. Guo gang. innovation design of carbon-free car based on TRIZ theory [J]. Chinese high-tech enterprises (a), 2015, (3): 31-32,33.

19. Du Lei, Ye Hai. Consumption pattern and stability analysis of carbon-free car [J]. Silicon Valley, 2013,6 (10): 72-73.

20. Zhao Peng-fei, Sun Ting-wei, Jia Yu-chao et al. Study on design and error compensation of carbon-free car $[\mathrm{J}]$. Science \& Technology Vision 2014, (14): 10-11.

21. Fan Suf-en. Turning Processing Countermeasure and Method of Small Diameter Stainless Steel Thread [J]. China modern educational equipment, 2015, (5):101-103. 